\title{
PROSPECTS OF APPLICATION OF COMPOSITE BIORESORABLE MATERIALS IN DENTISTRY (literature review)
}

Doctor of Medicine, Prof. Nigman Lukmanovich Khabilov *, Farhod Komiljonovich Usmanov *, Tulyaganov Jamshid Shovkatovich *,

Rakhimov Bakhtiyor Gafurjonovich. *

* Tashkent State Dental Institute

Contact person - FK Usmanov, tel. + 99890-900-12-30

Key words: dentistry, dental implants, composite materials, osseointegration, chitosan, biocompatibility, bioresorbability, antibacterial activity.

Annotation. The unique properties of Bombyx mori chitosan from silkworm pupae (TSh 88.2-13: 2011) (biocompatibility, bioresorbability, non-toxicity, antibacterial properties, hemostatic) will find wide application in therapeutic, surgical and orthopedic dentistry. The use of chitosan from local sources of raw materials will be in demand to create bioactive coatings on dental implants in domestic dentistry.

Summery. The unique properties of Bombyx mori chitosan from silkworm pupae (TSh 88.2-13: 2011) (biocompatibility, bioresorbability, non-toxicity, antibacterial properties, hemostatic) will find wide application in therapeutic, surgical and orthopedic dentistry. The use of chitosan from local sources of raw materials will be in demand to create bioactive coatings on dental implants in domestic dentistry.

Currently, active attempts are being made to strengthen osseointegration due to the inclusion of new generation composite bioresorbable materials in implants. One of the promising biomaterials for this was chitosan, which has a number of properties that can bring it to the forefront in prosthetics: it is non-toxic, has biocompatibility, bioresorbability and moderate antibacterial properties [1].

Chitosan is obtained from chitin and the possibilities of its production technology have not been fully exhausted yet.

The chains of the linear amino chitin amino polysaccharide are interconnected by hydrogen bonds. Each chain consists predominantly of repetitive $\mathrm{N}$-acetyl-amido-2-deoxy- $\beta$-D-glucose residues and significantly less of the glucosamine residues.

Both types of residues are connected in chains with ${ }^{\circ}(1 \rightarrow 4)$-glycosidic bonds (Fig. 1).

The pronounced chondro- and osteoinductive effects of three-dimensionally organized chitosan have been confirmed by numerous experiments [2, 3].

Technological aspects of creating such surfaces, as well as the results of their osseointegration in vivo, are currently being actively studied.

Prospects for the use of chitosan - implantology, tissue engineering and the development of tools for the delivery of drugs to organs and tissues. Chitosan is found in the outer skeleton of some crustaceans, the cuticle of insects, the cell wall of fungi and in plankton. 

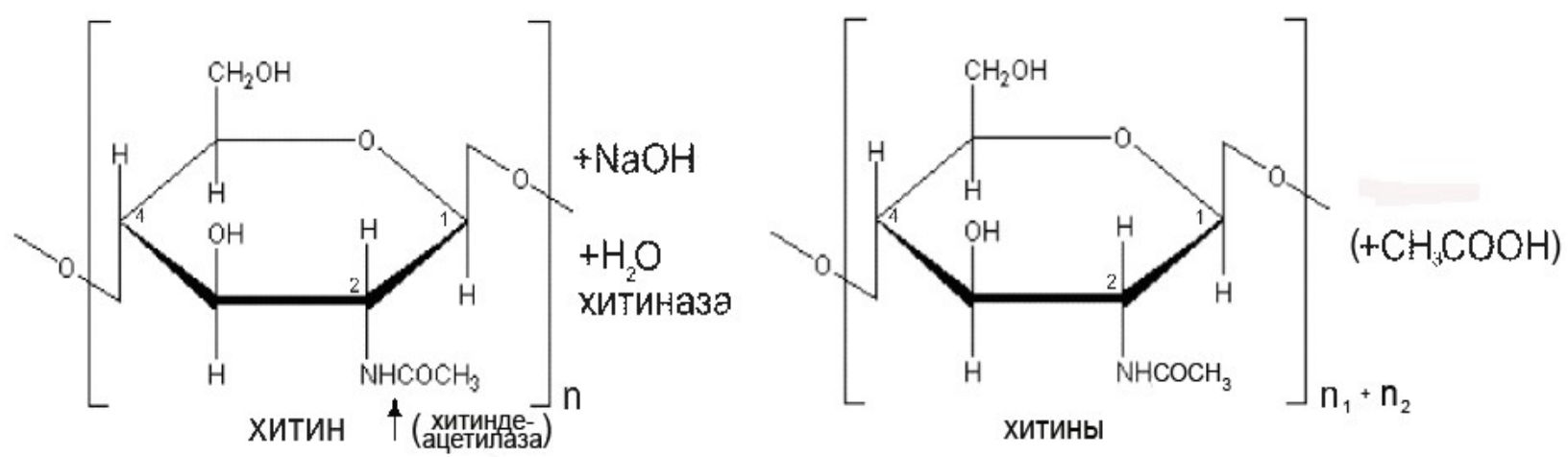

Pic.1. Depolymerization of chitin $\mathrm{n}$ with hydrolysis of a part of ${ }^{\circ}(1 \rightarrow 4)$ glycosidic bonds with the participation of alkali or hydrolytic chitinase enzyme with the formation of two polymers $n 1+n 2$. The arrow indicates the place of deacetylation by hydrolysis of the acetamide bond with chitin deacetylase to form instead of it the protonated amino group R-NH3 and acetic acid (in brackets).

\section{The use of chitosan and its derivatives in dentistry.}

A great contribution to the study of chitosan in dentistry was made by the Italians R. Muzzarelli et al. [4-7]. In particular, they used chitosan in combination with ascorbic acid in the treatment of generalized periodontitis [5]. By special treatment, a gel was obtained, which was injected into deep pockets after open curettage. 2 months after the treatment, the mobility of the teeth approached the norm, whereas prior to the treatment, the mobility of the II degree was clinically determined. The depth of the pathological pocket decreased, the level of epithelial attachment was restored.

Studies have been conducted to study the antibacterial activity of chitosan in the field of surgical dentistry under the guidance of S. Shomina. [eight]. The dissertation shows that representatives of conditionally pathogenic microflora are more sensitive to chitosan, while normal microflora is more stable. Chitosan has antimicrobial activity against pathogenic staphylococci, streptococci, enterobacteria, corynebacteria, micrococci, and Candida fungi. When using a photosensitizer based on chitosan and methylene blue and low-intensity laser radiation (LILR) in the infrared (IR) range, the number of viable bacteria sharply decreases by 7 orders and to zero in staphylococci and streptococci. The antiinflammatory effect and the healing effect of LLLT are enhanced. A single treatment of a purulent wound in the oral cavity according to the method described above in patients with acute purulent periostitis or the surface of the wound with abscesses and phlegmon reduced the number of pathogenic microbes. The frequency of excretion of microorganisms in the affected area decreased by 2 orders of magnitude. After treatment with chitosan, microorganisms did not show hemolytic, lecithinase, plasma coagulase, RNA az activity.

Chitosan enhances local immunity in the treatment of patients with acute purulent periostitis with a photosensitizer based on chitosan and methylene blue in the IR range. The level of lysozyme in the oral cavity increases by 3 times. In case of acute purulent periostitis of the maxillofacial area in polyclinic conditions, it is recommended to use for therapeutic purposes a $1 \%$ solution of chitosan on $0.25 \%$ 
$\mathrm{HCl}$ in combination with methylene blue and LILR. Treatment of acute purulent periostitis according to the indicated method improves the outcome of therapy, including the reduction of treatment time by 1-2 days compared with traditional methods of treatment. A complete cessation of the discharge and cleansing of the wound occurred, on average, at $4.3+/-0.8$ days after surgery, which is, on average, 2.9 days earlier than in patients treated by traditional methods.

Irradiation of LLLT is necessary at each bandaging after pre-cleaning of a purulent wound from necrotic tissue and treatment with chitosan solution in a complex with methylene blue.

A. Maygurov et al. (2006) used 2\% chitosan ascorbate gel (degree of deacetylation $95 \%$, MM 180-200 $\mathrm{kDa}$ ) and zinc oxide in a 1: 2 ratio in the treatment of deep caries [9]. There was no toxic effect on the pulp.

A high bacteriostatic effect due to the agglutination of microbes and a pronounced anti-inflammatory effect due to the activation of hyaluronidase and ${ }^{\circ}$ glucuronidase were noted. Three months after applying the paste, the dense obliteration of the dentinal tubules and the pronounced mineralization of reparative dentin were visible. Clinically there was no response to stimuli, the color of the tooth crown remained, the indices of electrical excitability came to normal.

In the study of the antibacterial activity of chitosan gel preparations on a mixed culture of bacteria isolated from root canals with destructive periodontitis, $8 \%$ chitosan gel had the most pronounced antibacterial effect. Bone tissue was completely restored after 12 months. in $62.2 \%$ of patients, partially recovered in $32.7 \%$ with a tendency to complete recovery in the long-term. The gel form $38 \%$ vodoratvorimogo ascorbate chitosan with metronidazole (MW 70kDa, deacetylation degree of $87 \%$, particle diameter of less than 160 microns) in the treatment of chronic catarrhal gingivitis facilitates rapid elimination of gingival inflammation, amplifies microvesicular transport of substances through the lumen of the capillaries, reduces edema, and restores structural gums organization .

In the treatment of chronic periodontitis of moderate severity, a sponge containing $8 \%$ chitosan ascorbate, $2 \%$ bovine collagen acetate, and metronidazole in a dose of $0.016 \mathrm{mg} / \mathrm{cm} 2$ was used. A sterile sponge with a size of $0.3 \times 0.3 \mathrm{~cm}$ was inserted into the periodontal pocket under a protective bandage once a day with an interval of 2 days. A decrease in bleeding of the gums was clinically noted, the mobility of the teeth decreased, and the painful sensation during the meal was stopped. The effect of anti-inflammatory action was $60.5 \%$. As a result of the research, a positive effect of chitosan was observed in various pathologies of the oral cavity.

A porous implant with chitosan and collagen, together with bone morphogenetic protein (BMP-7) and periodontal ligament cells, Zhang Y. et al. (2007) introduced a cannula defect in dogs. The formation of young bone was more intense in the experiment than in the control without chitosan, which was confirmed by laser confocal microscopy, an increase in the activity of alkaline phosphatase, a marker of osteoblasts, an increase in osteopontin and bone sialoprotein [10]. 
When using chitosan immobilized on a nanofibre membrane, it is complex with BMP-2 Park Y.Y. et al. (2006) obtained a significant osteoinductive effect [11].

During cystectomy surgery with resection of the root apex and removal of wisdom teeth, methylpyrolidinone chitosan was used to fill in bone defects, which was obtained as a sponge [4]. Histological and electromicroscopic examination of the removed tissues indicated that its use promotes the growth of capillaries, perivascular tissues and stimulation of mesenchymal cells.

In vivo studies have confirmed that methylpyrrolidinone chitosan is destroyed by the action of oral lysozyme. The resulting chitosan oligomers activate macrophages and stimulate the formation of collagen. Monomers resulting from degradation are used to rearrange glucosaminoglycans in the extracellular matrix to restore bone tissue. The osteoconductive properties of methylpyrolidinone chitosan were confirmed experimentally in rabbits, which are similar to the processes described above in humans [6]. Modification of chitosan by introducing an imidazole group, increased the cation ability of chitosan and increased its osteoinductive properties. The use of imidazole chitosan is much more effective than chitosan alone, and more effective than methyl pyrolidinone, in cystectomy with resection of the root apex and removal of wisdom teeth [6].

M. Ito (1991), using hydroxyapatite powder and $\mathrm{CaO}$ and $\mathrm{ZnO}$ additives with chitosan solution [12], obtained a rapidly hardening paste with high rates of compression. It is possible to adjust the compression by changing the percentage ratio of the components in the chitosan solution. The author notes a pronounced anti-inflammatory effect of the paste and the absence of migration of hydroxyapatite particles into the surrounding tissues.

R.Murugan, R.Ramakrishna (2004) used chitosan to increase the bioresorption of hydroxyapatite [13]. Composite hydroxyapatite-chitosan had good biocompatibility, bioresorbability, had a pronounced hemostatic effect, high antibacterial activity, plasticity and good adhesion. When processing carbonate apatite used $5-10 \%$ solution of chitosan. In the IR spectra of the composite, characteristic peaks for apatite carbonate are shown, while the structure of apatite carbonate is preserved. With an increase in chitosan concentration in solution, the bands at 603 and $571 \mathrm{~cm}-1$, which characterize the crystallinity of the structure, decrease. When studying the ratio between the content of $\mathrm{Ca} 2+$ and carbonate with apatite-chitosan in the model solution, it was found that the higher the concentration of chitosan, the greater the level of $\mathrm{Ca} 2+$, while reducing the amount of chitosan, the content of $\mathrm{Ca} 2+$ decreases. However, the parameters of the crystal lattice of apatite carbonate after treatment with chitosan remained almost unchanged. In the study of $\mathrm{pH}$ under the conditions of resorption of composites, it was found that the higher the concentration of chitosan in carbonate apatite, the lower the $\mathrm{pH}$. The $\mathrm{pH}$ level becomes unchanged at $\mathrm{pH}$ 7.1. The obtained nanocrystalline apatite carbonate from an aqueous solution at low temperature with the addition of chitosan can be used in the replacement of bone defects with the activation of bioresorption of apatite carbonate. 
R. Murugan et al. (2005) used chitosan to treat bovine-derived hydroxyapatite carbonate to improve solubility [14]. The authors noted that, depending on the concentration of chitosan in the solution, the rate of dissolution of hydroxyapatite carbonate in isotonic solution increased. A decrease in the $\mathrm{pH}$ of the solution for hydroxyapatite carbonate with a high chitosan content (from $\mathrm{pH}$ 7.4 to $\mathrm{pH} 7.1$ for 20 days) was observed, whereas with pure carbonate hydroxyapatite, the $\mathrm{pH}$ decreased slightly. Studies of the diffraction pattern revealed no changes in the crystal lattice of hydroxyapatite carbonate when interacting with chitosan. The IR spectra showed that with an increase in the content of chitosan in solution, the crystallinity of the hydroxyapatite carbonate structure decreases.

R. Tarsi et al. (1995) investigated the adsorption of S. mutans on the surface of hydroxyapatite granules in the presence of low-molecular chitosan and its derivatives $-\mathrm{N}$-carboxymethyl chitosan and imadazolyl chitosan [15]. As a control, saliva was used in the presence and without sucrose. The authors showed that the treatment of hydroxyapatite granules with chitosan and its derivatives significantly reduces the adhesion of S.mutans. The presence of chitosan in toothpaste, chewing gum and prophylactic rinse liquids significantly reduces the colonization of S. mutans on the surface of hydroxyapatite. Stop the growth of pathogenic flora due by agglutination of microbial bodies with chitosan. The agglutination mechanism is identical to the gluing of red blood cells by polycations. Binding of chitosan to the sugar receptors on the cell membrane provides a bacteriostatic effect [16].

Chitosan has been used in dental surgery for the treatment of fractures, distraction osteogenesis, when calcium phosphate, sulphate cements were introduced into the composition [17], HA pastes [18], c $\beta$-TCPF [19]; treatment of osteomyelitis (Aimin et al. 1999), osteoporosis (Hi et al. 2007). All researchers noted a positive effect.

In maxillofacial implantology, when coating titanium implants, chitosan contributed to accelerated osseointegration, reduced edema, an inflammatory component $[20,21]$.

Thus, a review of the literature indicates a significant interest in chitosan by foreign and domestic researchers.

In the global industry, the production of chitin and chitosan has field crustacean crustaceans as a raw material base, but the volumes of this production are limited to catch volumes. Silk production has traditionally been developed in Uzbekistan, and the waste of the silkworm Bombyx mori is the raw material source for the isolation of a very valuable natural polysaccharide, chitin, a modification of which synthesizes a second, no less demanded product, chitosan [22].

For the first time, a technology has been developed on the basis of the NCCPP and the production of this polymer has been mastered, there are approved specifications for Chitosan from silkworm pupae, TS 88.2-13: 2011, and a trademark for Bombyx mori - Chitosilk is obtained. Chitosan Bombyx mori is a low-toxic substance and in its action belongs to the $\mathrm{V}$ class of toxicity. In addition, the sensitivity of chitosan Bombyx mori to microorganisms St.saprofiticus, Str.pyogens, Ent.faecalis, Esch. Coli LP, Esch. Coli LN, Prot.vulgaris, Klebsiella, 
Actinomycete in vitro [23]. It is revealed that $\mathrm{HZ}$ has an effect on both grampositive and gram-negative flora, which will allow its use in dental practice. The unique properties of chitosan (biocompatibility, bioresorbability, non-toxicity, antibacterial properties, hemostaticity) will be widely used in therapeutic, surgical and orthopedic dentistry. The use of Bombyx mori chitosan from local sources of raw materials will be in demand to create bioactive coatings on dental implants in domestic dentistry.

\section{LITERATURE}

1. Gaifullin, N.M. Recombinant bone morphogenetic protein 2 stimulates the remodeling of chitosan-based porous scaffold into hyaline-like cartilage: study in heterotopic implantation // European Journal of Molecular Biotechnology. -2013. № 1 (1). - P. 11-14.

2. Lyabin, M. P. Improving the technology of chitosan production // Bulletin of Volgograd State University. Series 11: Natural Sciences. - 2011. - №2 (2). Pp. 1722.

3. Chang, P. C. Evaluation of functional dynamics during osseointegration and regeneration associated with oral implants: a review / R. Chang // Clin. Oral Implants Res. - 2010. - № 21 (1). - P. 1-12

4. Muzzarelli R., Biagini G. et al. Osteoconduction exerted by methylpyrrolidinone chitosan used in dental surgery // Biomaterials. - 1993. - Vol.14. - №1. - P. 39-43.

5. Muzzarelli R., Biagini G. et al. Reconstruction of paradontal tissue with chitosan // Biomaterials. - 1989. - Vol.10. - №11. - P. 598-603.

6. R. Muzzarelli, G. Biagini et al. Osteoconductive properties of methylpyrrolidinone chitosan in an animal model // Biomaterials. - 1993. - Vol.14. - №12. - P. 925-929.

7. Muzzarelli R., Baldassarre V. et al. Biological activity of chitosan: ultrastructural study // Biomaterials. - 1988. - Vol.9. - №5. - P. 247-252.

8. Shomina S.A. The use of chitosan in the treatment of acute inflammatory diseases of the maxillofacial area. Diss. Cand. honey. sciences. - Tver. - 2002. $195 \mathrm{~s}$.

9. Maigurov A., Solntsev A., Bolshakov I. et al. The use of chitosan in the treatment of inflammatory diseases of the oral cavity. Modern perspectives in the study of chitin and chitosan. Proceedings of the VIII international conference. - Ed. "VNIRO". - M. - 2006. - S.224-227.

10. Zhang Y., Song J., Shi B. et al. Combination of scaffold and adenovirus vector expressing bone regeneration at dental implant defects // Biomaterials. - 2007. Vol.28. -N.31.- P.4635-4642.

11. Park Y.J., Kim K.H., Lee J.Y. et al. Immobilization of bone morphogenetic protein-2 on a nanofibrous chitosan membrane for enhanced guided bone regeneration // Biotechnol. Appl.Biochem. - 2006. - Vol.43 - Pt.1 - P.17-24.

12. Ito M. In vitro properties of a chitosan-bonded hydroxyapatite bone-filling paste // Biomaterials. - 1991. - Vol.12. - №1. - P. 41-45.

13. Murugan R., Ramakrishna R. Bioresorbable composite bone paste using polysaccharide based nanohydroxyapatite // Biomaterials. - 2004. - Vol.25. - p. 3829-3835. 
14. Murugan R., Kumar S. et al. Hydroxyl carbonate apatite hybrid bone composites using carbohydrate polymer // J. of Composite Materials. - 2005. Vol.39. - №13. - P.1159-1166.

15. Tarsi R., Muzzarelli R. Guzman C. et al. Inhibition of Streptococus mutans. Adsorption of hydroxyapatite by low-molecular weight chitosans // J. Dent. Research. - 199

16. Velichkov A.D., Nikolova S.F., Vejanov D.K. // C.R. Acfd. Bulg. Sci. - 1989. Vol.42. -№3.- P.81-84. (cited according to the number 10 Gorovoy L., Kosyakov V.) 7. - Vol.76. -№ 2. - P. 665-672.

17. Cho B.C., Park J.W., Baik B.S. et al. It is a role of hyaluronic acid, chitosan and calcium sulfate. J. Craniofac. Surg. - 2002. - Vol.13. - P.783-793.

18. Kawakami, T., Antoh, M., Hasegawa, H. et al. Experimental study on osteoconductive properties of a chitosan-bonded hydroxyapatite self-hardening paste // Biomaterials. - 1992. -Vol.13. - P.759-763.

19. Yin Y., Ye F., Cui J. et al. Preparation and characterization of macroporous chitosan gelatin / beta-tricalcium phosphate composite scaffolds for bone tissue engineering // J. Biomed.Mater. Res. - 2003. - Vol.63A. - P.844-855.

20. Bumgardner J.D., Wiser R., Elder S.H. et al. Contact angle, protein adsorption and osteoblast precursor cell attachment to chitosan coatings bonded to titanium // J. Biomater. Sci. Polym ed. - 2003. - Vol.14. - 1401-9.

21. Wang X., Ma J., Wang Y., He B. Bone repair in radii and tibias of rabbits with phosphorylated chitosan reinforced calcium phosphate cements // Biomaterials. 2002. Vol.23. - P.4167-76.

22. Rashidova S.Sh., Milusheva R.Yu. "Chitin and chitosan Bombyx mori. Synthesis, properties and application ", Tashkent, FAN Publishing House, 2009, p.246. 